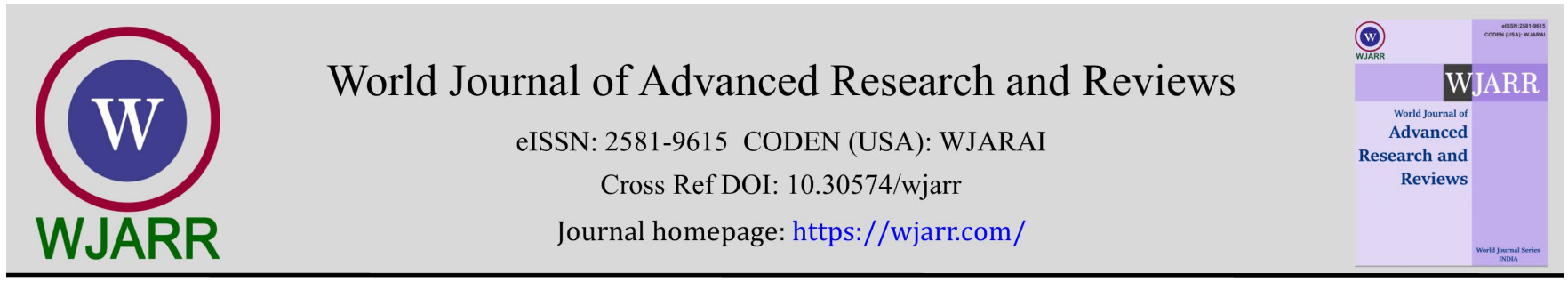

(RESEARCH ARTiClE)

Check for updates

\title{
Evaluation of hepatic fibrosis in patients with chronic hepatitis B virus by non- invasive methods: Aspartate-to-platelet ratio index, Fibrosis-4, fibrotest and fibroscan
}

Laté Mawuli Lawson-Ananissoh ${ }^{1,}{ }^{*}$, Aklesso Bagny ${ }^{1}$, Oumboma Bouglouga ${ }^{2}$, Laconi Kaaga ${ }^{1}$, Gad Namdiro ${ }^{1}$, Rafiou El-Hadji Yakoubou ${ }^{2}$, Lidawu Roland-Moise Kogoe ${ }^{1}$ and Debehoma Venceslas Redah ${ }^{1}$

${ }^{1}$ Hepato-Gastroenterology unit, Campus Teaching Hospital, Lome-Togo.

${ }^{2}$ Hepato-Gastroenterology unit, Kara Teaching Hospital, Kara-Togo.

World Journal of Advanced Research and Reviews, 2022, 13(01), 172-179

Publication history: Received on 30 November 2021; revised on 04 January 2022; accepted on 06 January 2022

Article DOI: https://doi.org/10.30574/wjarr.2022.13.1.0767

\begin{abstract}
Background: The process of hepatic fibrosis is common to the various etiologies of chronic liver disease such as viral hepatitis B.
\end{abstract}

Objective: To evaluate hepatic fibrosis by non-invasive markers such as Aspartate-to-Platelet Ratio Index (APRI), fibrosis-4 (FIB-4), fibrotest and fibroscan.

Patients and Method: This was a descriptive study during a period of 32 months. Included in our study were the records of outpatients, chronic carriers of hepatitis B virus without viral co-infection C, D or HIV, followed in the Gastroenterology unit of the Campus Teaching Hospital of Lome-Togo.

Results: We retained 222 patients. Among the patients, 148 patients (66.67\%) were classified in Phase 3 (inactive carrying). Only 10 patients (4.50\%) had a APRI score indicating a fibrosis stage $\geq$ F4 (presence of cirrhosis). A FIB-4 score indicating the presence of cirrhosis was found in 12 patients (5.40\%). The most represented stage at fibrotest was the F0 stage (45.45\%). Cirrhosis was noted in $6.06 \%$ of cases at fibroscan. Patients with APRI score $\leq 2(96.23 \%)$ had a FIB-4 score $\leq 3.25,(\mathrm{p}=0.0088)$.

Conclusion: The evaluation of hepatic fibrosis during chronic hepatopathies is essential for patients care because it influences therapeutic decisions.

Keywords: Hepatic fibrosis; APRI; FIB-4; Fibrotest; Fibroscan; Togo

\section{Introduction}

Viral hepatitis B (VHB) is a major public health problem. Complications of chronic VHB cause 1.1 million deaths each year worldwide [1] and represents 5 to $10 \%$ of the causes of liver transplantation [1]. Sub-Saharan Africa is a highly endemic area with a high prevalence of $6.1 \%$ of the adult population infected [1]. The process of hepatic fibrosis is common to the various etiologies of chronic liver disease and determines the occurrence of complications such as portal hypertension, liver failure and hepatocellular carcinoma, clinical situations involving life-threatening prognosis [2, 3]. The evaluation of hepatic fibrosis during chronic liver disease is useful in the management of patients because it conditions therapeutic decisions and screening for complications [3]. It is also a marker for the progression of liver

\footnotetext{
* Corresponding author: Laté Mawuli Lawson-Ananissoh

Hepato-Gastroenterology unit, Campus Teaching Hospital, Lome-Togo.
}

Copyright ( 2022 Author(s) retain the copyright of this article. This article is published under the terms of the Creative Commons Attribution Liscense 4.0 
disease [3]. If the reference examination remains hepatic biopsy, its invasiveness, high cost and inconsistent acceptability by patients, have motivated the development of new methods of evaluating fibrosis, called « non-invasive » tests. Many studies [4-10] including a few african studies [11,12] have led to proposing and then validating noninvasive tests capable of replacing liver biopsy to estimate the degree of fibrosis in patients with chro nic hepatitis B and $C$ viruses. In Togo, the prevalence of hepatits $B$ virus (HBV) varies between $5 \%$ and $16.4 \%[13$, 14]. Despite this high prevalence, there is still no national program to combat HBV. Similarly, no studies have been conducted on the evaluation of hepatic fibrosis in subjects with chronic hepatitis B. The purpose of this study was to evaluate hepatic fibrosis by non-invasive markers such as Aspartate-to-Platelet Ratio Index (APRI), fibrosis-4 (FIB-4), fibrotest and fibroscan in patients with chronic HBV.

\section{Patients and method}

\subsection{Type and period of study}

This was a descriptive study with retrospective data collection, from January 1, 2016 to December 31, 2017 (24 months), and prospective data collection, from January 1 to August 31, 2018 (08 months), a 32 months period.

\subsection{Study population}

Our study population consisted of all patients of both sexes, aged 15 years and over, chronically infected with hepatitis B virus, followed as an outpatient in the Hepato-gastroenterology department of the Campus Teaching Hospital of LomeTogo.

\subsection{Inclusion criteria}

Included in our study were the records of patients with chronic VHB without viral co-infection C, D or HIV, in which the following parameters were available: age, hemogram, a transaminase assay with or without fibrotest and / or fibroscan.

\subsection{Criteria for non-inclusion}

Not included in our study:

- $\quad$ Patients who did not have a minimal check-up including: a hemogram; transaminases; viral markers B (HBsAg, total anti-HBcAb, HBeAg, anti-HBeAb, viral load B).

- $\quad$ Patients with autoimmune hepatitis and all alcoholic patients with daily alcohol consumption $\geq 50 \mathrm{~g} / \mathrm{l}$ in men and $\geq 40 \mathrm{~g} / \mathrm{l}$ in women.

- $\quad$ patients with decompensated or degenerated viral cirrhosis B.

\subsection{Definition of the different phases of chronic hepatitis B in our study}

We distinguished 04 chronic conditions of the disease in our study [15]:

- Phase 1: HBeAg-positive chronic HBV infection, previously termed "immune tolerant" phase; characterised by the presence of serum HBeAg, very high levels of HBV DNA and ALT persistently within the normal range. In the liver, there is minimal or no liver necroinflammation or fibrosis but a high level of HBV DNA ( $>10^{7}$ IU $\left./ \mathrm{ml}\right)$

- $\quad$ Phase 2: HBeAg-positive chronic hepatitis B is characterised by the presence of serum HBeAg, high levels of HBV DNA and elevated ALT. In the liver, there is moderate or severe liver necroinflammation and accelerated progression of fibrosis.

- $\quad$ Phase 3: HBeAg-negative chronic HBV infection, previously termed 'inactive carrier' phase, is characterised by the presence of serum antibodies to HBeAg (anti-HBe), undetectable or low $(<2,000 \mathrm{IU} / \mathrm{ml}) \mathrm{HBV}$ DNA levels and normal ALT. Some patients in this phase, however, may have HBV DNA levels $>2,000$ IU/ml (usually $<20,000 \mathrm{IU} / \mathrm{ml}$ ) accompanied by persistently normal ALT and only minimal hepatic necro-inflammatory activity and low fibrosis.

- $\quad$ Phase 4: HBeAg-negative chronic hepatitis B previously termed immune reactivation phase, is characterised by the lack of serum HBeAg usually with detectable anti-HBe, and persistent or fluctuating moderate to high levels of serum HBV DNA (often lower than in HBeAg-positive patients), as well as fluctuating or persistently elevated ALT values. The liver histology shows necroinflammation and fibrosis. 


\subsection{Study conduct}

We conducted the interview in the consultation room and then conducted a complete physical examination. Paraclinical examinations were then requested. Patients had the choice to carry out these tests in the laboratory of the University Hospital-Campus, when these tests are available, in the laboratory of the national institute of hygiene or in a private laboratory. For the fibrotest, all the samples were taken in a private laboratory and sent to the CERBA laboratory in France. For viral load B, the samples were sent to the BIOLIM laboratory located within the Faculty of Health Sciences of the University of Lomé (FSS-UL) or to a private laboratory, a partner of the CERBA laboratory in France where they are sent. We received technical support from COCHIN Hospital (France) for the realization of fibroscan. The examination was performed in a room equipped with an examination table, using a portable fibroscan of FibroScan ${ }^{\circledR}$ Echosens 430 Mini type according to the recommended technique. The device was made available to the service for a period of 05 working days.

\subsection{Evaluation of hepatic fibrosis}

For the 4 non-invasive methods of evaluating the hepatic fibrosis, we have adopted in this study, the threshold values for interpretation recommended by World Health Organization (WHO). [1]

\subsection{Aspartate-to-platelet ratio index (APRI) [1]:}

- $\quad$ APRI $\leq 2$ : absence of cirrhosis (METAVIR score $<$ F4)

- $\quad$ APRI $>2$ : presence of cirrhosis (METAVIR score $\geq F 4$ )

\subsection{Fibrosis-4 (FIB-4) [1]:}

- $\quad$ FIB- $4 \leq 3.25$ : absence of cirrhosis (METAVIR score $<$ F4)

- $\quad$ FIB-4> 3.25: presence of cirrhosis (METAVIR score $\geq$ F4)

\subsection{Fibrotest:}

The interpretation thresholds [2] related to the METAVIR score and published by the company marketing the FibroTest ${ }^{\circledR}$ make it possible to group the different stages as follows:

- $\quad$ Minimal fibrosis (F0, F1) ;

- $\quad$ Clinically significant fibrosis ( $\geq$ F2) ;

- $\quad$ Severe fibrosis ( $\geq$ F3);

- $\quad$ Cirrhosis $(\geq \mathrm{F} 4)$

\subsection{Fibroscan or hepatic elastometry}

The success rate of the test is the ratio of the number of measurements validated by the device to the total number of measurements performed during the same test. The median value of the validated measurements is representative of the elasticity of the liver. The interquartile range (IQR) represents the interval around the median containing $50 \%$ of the valid measurements. To be considered interpretable and valid, the examination must include at least 10 measures with a success rate of at least $60 \%$ and the IQR / median must not exceed $30 \%$ of the examination result. The interpretation threshold values are [1]

- $\quad$ Minimal fibrosis (F0, F1): $<7 \mathrm{kPa}$

- Clinically significant fibrosis $(\geq \mathrm{F} 2): \geq 7 \mathrm{kPa}$

- $\quad$ Cirrhosis $(\geq \mathrm{F} 4):>11 \mathrm{kPa}$

\subsection{Data collection, entry and analysis}

APRI and FIB-4 scores were calculated using the Medicalcul software. The data collected were entered on the EpiData software version 3.1. The data entered were reconciled and then analyzed with the R Studio 3.4.4 software. The statistical analysis consisted of a descriptive analysis of the population and then a comparative analysis of the APRI, FIB4, fibrotest and fibroscan scores. In the descriptive analysis, for the characteristics collected, the results were presented in terms of size and frequency for the qualitative variables and, in terms of mean and standard deviation for the quantitative variables. In the comparative analysis, the statistical tests used were the Chi-2 and Fisher tests for comparing qualitative variables, and the Student test for quantitative variables. The significant threshold was 0.05. 


\subsection{Ethical considerations}

We had obtained an authorization from the direction of the University Hospital-Campus to freely carry out our study. We then met with the patients to be included in the study to explain the interest and the purpose of the study. All patients included in our study gave their free and informed oral consent for the use of their data. The collection, entry and processing of data had been done with strict respect for confidentiality and anonymity.

\section{Results}

\subsection{Global data}

During the study period, 222 patients were retained. Only 77 (34.68\%) were able to perform the fibrotest and 33 patients $(14.86 \%)$ received the fibroscan examination. Nine patients performed all the non-invasive methods tested (APRI, FIB-4, fibrotest and fibroscan). There was a male predominance (63.96\%) with a sex ratio of 0.56 female / male. The average age of the patients was 33.74 +/- 10.39 years (extremes, 18-72 years). The most represented age group was 30 to 40 years. The most common reason for consultation was the discovery of HBsAg during screening or routine check-up (92.354\%). Jaundice was found in 04 patients (1.8\%). The average AST level was $40.82 \pm 5.65$ IU / l (extremes of 12 and $228 \mathrm{IU} / \mathrm{l}$ ). The average ALT level was 47.89 $44.94 \mathrm{IU} / \mathrm{l}$ (extremes of 13 and $295 \mathrm{IU} / \mathrm{l}$ ). Beta-gamma block was found in 07 patients (3.15\%). Thrombocytopenia was noted in 43 patients (19.37\%). Thirty-three patients $(14.86 \%)$ had a positive HBeAg assay and 189 patients $(85.14 \%)$ had a positive anti-HBe Ac assay. The average viral load was $30761507.78 \pm 6624.17633 \mathrm{IU} / \mathrm{ml}$, ie $7.48 \pm 3.82 \mathrm{log}$ with extremes of $10 \mathrm{IU} / \mathrm{ml}$ and 1,000,000 IU / ml (ie 1 $\log$ and $9 \mathrm{log}$ ). Hepatomegaly was noted in 32 patients $(14.41 \%)$ on abdominal ultrasound.

\subsection{Chronic hepatitis B phases}

Among the patients, 148 patients (66.67\%) were classified in Phase 3 (figure 1).

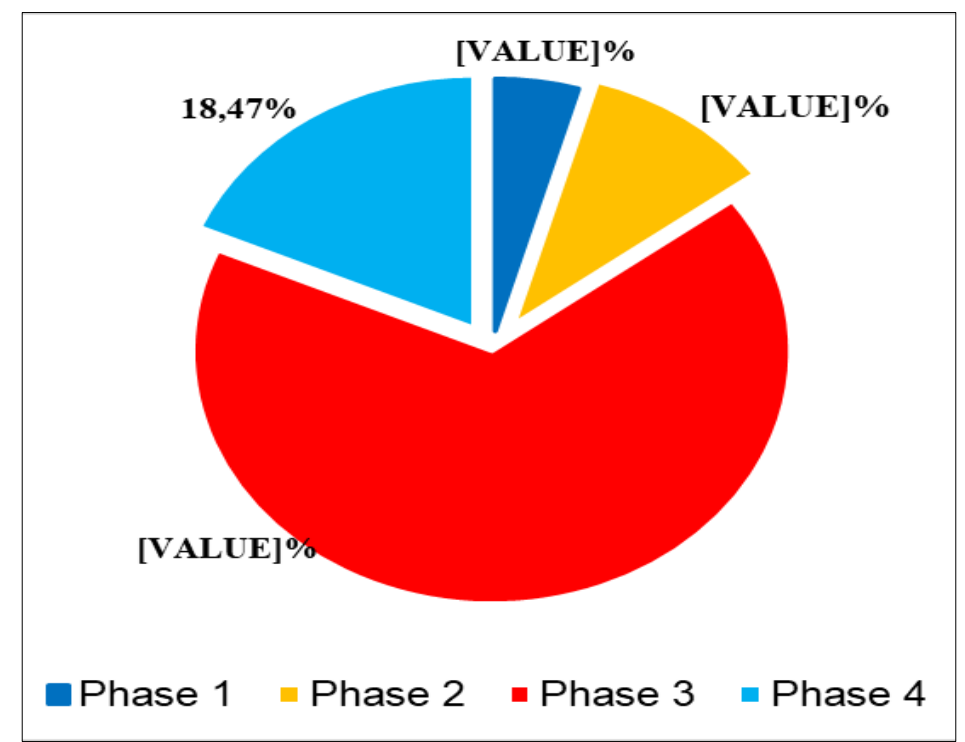

Phase 1 : HBeAg-positive chronic HBV infection; Phase 2 - HBeAg-positive chronic hepatitis B ; Phase 3: HBeAg-negative chronic HBV infection; Phase 4 : HBeAg-negative chronic hepatitis $B$

Figure 1 Distribution of patients according to the chronicity phase

\subsection{Assessment parameters for hepatic fibrosis (APRI, FIB-4, fibrotest, fibroscan)}

The table 1 summarizes the characteristics of the parameters for assessing hepatic fibrosis (APRI, FIB-4, fibrotest, fibroscan). With fibrotest, significant fibrosis is noted in $20.78 \%$. With fibroscan, the minimal fibrosis was found in $78.79 \%$ followed by significant fibrosis ( $\geq$ F2) in 05 patients $(15.15 \%)$. 
Table 1 Characteristics of the evaluation parameters for hepatic fibrosis

\begin{tabular}{|l|c|c|c|}
\hline & Mean \pm Standard deviation & Extremes & Cirrhosis n (\%) \\
\hline APRI $(\mathrm{N}=222)$ & $0.65 \pm 1.09$ & $0.15-4.16$ & $10(4.5)$ \\
\hline FIB-4 $(\mathrm{N}=222)$ & $1.25 \pm 4.15$ & $0.3-6.27$ & $12(5.4)$ \\
\hline Fibrotest $(\mathrm{N}=77)$ & $0.31 \pm 0.36$ & $0.01-0.89$ & $6(7.79)$ \\
\hline Fibroscan $(\mathrm{N}=33)$ & $6.33 \pm 1.97$ & $3.5-12.3$ & $2(6.06)$ \\
\hline
\end{tabular}

\subsection{Comparative analysis of scores}

\subsubsection{APRI score and other parameters}

Patients with APRI score $\leq 2$ had a FIB-4 score $\leq 3.25$, or $96.23 \%$ ( $=0.0088$ ); patients with APRI score $\leq 2$ had a fibrotest score $<\mathrm{F} 4=93.33 \%(\mathrm{p}=0.1507)$ as shown in Table 2. Patients with an APRI score $\leq 2 \mathrm{had}$ a fibroscan $\leq 11 \mathrm{kPa}$, or $96.77 \%(\mathrm{p}=0.1133)$.

Table 2 Relationship between APRI and FIB-4, fibrotest, fibroscan, chronicity phases

\begin{tabular}{|c|c|c|c|c|c|c|c|}
\hline & \multicolumn{2}{|c|}{$\begin{array}{l}\text { APRI } \leq 2 \\
(\mathrm{~N}=212)\end{array}$} & \multicolumn{2}{|c|}{$\begin{array}{c}\text { APRI > } 2 \\
(\mathrm{~N}=10)\end{array}$} & \multicolumn{2}{|c|}{$\begin{array}{c}\text { Total } \\
(\mathrm{N}=222)\end{array}$} & \multirow[t]{2}{*}{ p-value } \\
\hline & $\mathbf{n}$ & $(\%)$ & $\mathbf{n}$ & $(\%)$ & $\mathbf{n}$ & $(\%)$ & \\
\hline FIB-4 $(\mathrm{N}=222)$ & & & & & & & $0.0088^{+}$ \\
\hline$\leq 3.25$ & 204 & 96.23 & 6 & 60.00 & 210 & 94.60 & \\
\hline$>3.25$ & 8 & 3.77 & 4 & 40.00 & 12 & 5.40 & \\
\hline Fibrotest (N=77) & & & & & & & $0.1507^{++}$ \\
\hline$<\mathrm{F} 4$ & 70 & 93.33 & 1 & 50.00 & 71 & 92.21 & \\
\hline$\geq \mathrm{F} 4$ & 5 & 6.67 & 1 & 50.00 & 6 & 7.79 & \\
\hline Fibroscan $(\mathrm{N}=33)$ & & & & & & & $0.1193^{++}$ \\
\hline$\leq 11 \mathrm{kPa}$ & 30 & 96.77 & 1 & 50.00 & 31 & 93,94 & \\
\hline$>11 \mathrm{kPa}$ & 1 & 3.23 & 1 & 50.00 & 2 & 6.06 & \\
\hline Chronicity phases & & & & & & & $<0.0001^{++}$ \\
\hline 1 & 10 & 4.72 & 0 & 0.00 & 10 & 4.50 & \\
\hline 2 & 20 & 9.43 & 3 & 30.00 & 23 & 10.36 & \\
\hline 3 & 148 & 69.81 & 0 & 0.00 & 148 & 60.67 & \\
\hline 4 & 34 & 16.04 & 7 & 7.00 & 41 & 18.47 & \\
\hline
\end{tabular}

\subsection{FIB-4 score and other parameters:}

Patients with FIB-4 score $\leq 3.25$ had a fibrotest score $<F 4=93.33 \%$ with $p=0.1507$; patients with FIB-4 score $\leq 3.25$ had a fibroscan $\leq 11 \mathrm{kPa}$, or $93.75 \%(\mathrm{p}=0.9999)$ as shown in Table 3 .

\subsection{Fibrotest and fibroscan}

Patients with fibroscan $\leq 11 \mathrm{kPa}$ had a fibrotest $<\mathrm{F} 4,77.78 \%(\mathrm{p}=0.9999)$.

\subsection{Relationship between chronicity phases and scores}

Patients classified in phase $2(30 \%)$ and phase $4(70 \%)$ had an APRI score $>2$ (cirrhosis) with $\mathrm{p}<0.0001$ (Table 2). Patients classified in phase 4 (41.67\%) had a FIB-4 score $>3.25$ (cirrhosis) with $p=0.0362$ (Table 3). Patients classified in phase $2(50 \%)$ and phase $4(50 \%)$ had fibroscan cirrhosis with $\mathrm{p}=0.0682$. 
Table 3 Relationship between FIB-4 and fibrotest, fibroscan, chronicity phases

\begin{tabular}{|l|c|c|c|c|c|c|c|}
\hline & \multicolumn{2}{|c|}{$\begin{array}{c}\text { FIB-4 3.25 } \\
\text { (N=75) }\end{array}$} & \multicolumn{2}{c|}{$\begin{array}{c}\text { FIB-4 } \\
\text { (N=2) }\end{array}$} & \multicolumn{2}{c|}{$\begin{array}{c}\text { Total } \\
\text { (N=77) }\end{array}$} & p-value \\
\hline & $\mathbf{n}$ & $\mathbf{( \% )}$ & $\mathbf{n}$ & $\mathbf{( \% )}$ & $\mathbf{n}$ & $\mathbf{\%}$ & \\
\hline Fibrotest (N=77) & & & & & & & $0.1507^{++}$ \\
\hline$<\mathrm{F} 4$ & 70 & 93.33 & 1 & 50.00 & 71 & 92.21 & \\
\hline$\geq \mathrm{F} 4$ & 5 & 6.67 & 1 & 50.00 & 6 & 7.79 & \\
\hline Fibroscan (N=33) & & & & & & & $0.9999^{++}$ \\
\hline$\leq 11 \mathrm{kPa}$ & 30 & 93.75 & 1 & 100.00 & 31 & 93.94 & \\
\hline$>11 \mathrm{kPa}$ & 2 & 6.25 & 0 & 0.00 & 2 & 6.06 & \\
\hline Chronicity phases & & & & & & & $0.0362^{++}$ \\
\hline 1 & 9 & 4.29 & 1 & 8,33 & 10 & 4.50 & \\
\hline 2 & 21 & 10.00 & 2 & 16.67 & 23 & 10.36 & \\
\hline 3 & 144 & 68.57 & 4 & 33.33 & 148 & 66.67 & \\
\hline 4 & 36 & 17.14 & 5 & 41.67 & 41 & 18.47 & \\
\hline
\end{tabular}

\section{Discussion}

In our study, the evaluation of hepatic fibrosis was made only by non-invasive methods, with no possibility of comparison between the latter and the hepatic puncture-biopsy which is the reference test. In most of the reported studies $[6-8,11,12]$, the comparison between the reference examination and each non-invasive method tested was the rule, which allowed the diagnostic performance of the latter to be determined.

\subsection{The APRI score}

In Mahassadi's study [11] in Côte d'Ivoire, the average APRI score was 0.72 (IQR = 0.4-1.85). For Bonnard [12] in Burkina Faso, with an APRI score threshold value of 1.0, significant fibrosis could be correctly predicted with a sensitivity of $55 \%$ and a specificity of $50 \%$. Similarly, with a threshold value of 1.2 , cirrhosis could be correctly excluded with a sensitivity of $55 \%$ and a specificity of $51 \%$. These results noted by the various authors show that the APRI score, a simple index using readily available laboratory results, can identify the presence or absence of cirrhosis with a reliable degree of accuracy. However, our study does not confirm this accuracy.

\subsection{The FIB-4 score}

In Mallet's study [7], a threshold value $\leq 1.45$ of the FIB-4 score differentiated moderate fibrosis from severe fibrosis with a sensitivity of $71.1 \%$ and a specificity of $73.1 \%$. Above 1.45 , the FIB-4 score was not informative. In that of Mahassadi [11] in Côte d'Ivoire, the average FIB-4 score was 1.35 (IQR = 0.78-2.20). For Bonnard [12] in Burkina Faso, with a FIB-4 score threshold value of 0.8 , significant fibrosis could be correctly predicted with a sensitivity of $74 \%$ and a specificity of $60 \%$; with a threshold value of 1.05 cirrhosis could be excluded with a sensitivity of $92 \%$ and a specificity of $70 \%$. These results show that the FIB-4 score is reliable for determining the presence or absence of cirrhosis. But our study does not confirm them.

\subsection{Fibrotest}

For Bonnard [12] in Burkina Faso, the group of patients with significant fibrosis had an average fibrotest score of 0.37 with a sensitivity of $77 \%$ and a specificity of $80 \%$. These results noted in the literature show that fibrotest correctly identifies significant fibrosis, severe fibrosis and cirrhosis. However, these results cannot be confirmed by our study.

\subsection{Fibroscan}

In the Lupşor study [8], with a threshold value of $7.4 \mathrm{kPa}$, significant fibrosis could be correctly predicted with a sensitivity of $76 \%$ and a specificity of $83.6 \%$. Similarly, with a threshold value of $11.8 \mathrm{kPa}$, cirrhosis could be correctly excluded with a sensitivity of $87 \%$ and a specificity of $90.8 \%$. For Bonnard [12] in Burkina Faso, with a threshold value of $7.3 \mathrm{kPa}$, significant fibrosis could be correctly predicted with a sensitivity of $75 \%$ and a specificity of $85 \%$. These 
results show that fibroscan is effective in detecting clinically significant fibrosis and cirrhosis. However, our study cannot confirm these results because of the small size of the patients who performed the fibroscan.

\subsection{APRI score and other parameters}

In the Mahassadi study [11]in Côte d'Ivoire, with an APRI score threshold value > 1.1, significant fibrosis could be correctly excluded in 61 (52\%) of 117 patients with a negative predictive value of $78.2 \%$. Similar results were obtained with the FIB-4 score (threshold value> 2.1) which correctly identified a significant absence of fibrosis in 62 (53\%) of the 117 patients. Nevertheless, the number of false negative patients was smaller with the FIB-4 score than with the APRI score (8.5\% and 14.5\% respectively). The APRI and FIB-4 scores had a maximum sensitivity of $94.4 \%$ and $88.9 \%$ respectively. Of the 18 patients with histological evidence of cirrhosis, 17 (94.4\%) were well classified and 1 (1.9\%) was a false negative. WHO recommends APRI and FIB-4 scores as non-invasive tests to evaluate the presence of cirrhosis in resource-limited environments [1]. There was no significant association between APRI and fibrotest on the one hand and between APRI and fibroscan on the other hand. This could be explained by the relatively small sample size of patients who performed fibrotest or fibroscan.

\subsection{FIB-4 score and other parameters}

In Mallet's study [7], FIB-4 score correctly identify patients with zero or moderate fibrosis with an AUROC of 0.81 (p $<0.001$ ) and was correlated with fibrotest in $89 \%$ of cases (correlation coefficient $=0.27, \mathrm{p}<0.001$ ) to exclude severe fibrosis. These results show that the FIB-4 score is close to that of the fibrotest for the prediction of significant fibrosis and cirrhosis. However, our study does not confirm these results. The association between FIB-4 and fibroscan was not significant; this could be explained by the relatively small sample size of patients who performed fibroscan.

\subsection{Fibrotest and fibroscan}

In our study, there was no association between fibrotest and fibroscan. WHO recommends fibroscan or fibrotest as a non-invasive test to detect hepatic fibrosis in settings where it is available and where cost is not a major barrier [1].

\subsection{Relationship between chronicity phases and scores}

In our study, there was a statistically significant relationship between chronicity phases and APRI score: patients classified as phase 1 (immune tolerance) and phase 3 (inactive carrying) of chronicity were more likely to have no cirrhosis according to the APRI score $(\mathrm{p}=0.0001)$; inactive carrying only exceptionally evolves towards cirrhosis. There was also a statistically significant relationship between chronicity phases and the FIB-4 score: patients classified as phase 3 chronicity (inactive carrying) were more likely to have no cirrhosis according to the FIB-4 score ( $p=0.0362$ ). This is in accordance with the literature [15]. There was no statistically significant relationship between the chronicity phases and the fibrotest nor with the fibroscan; this could be explained by the relatively small sample size of patients who performed fibrotest or fibroscan.

\section{Conclusion}

The evaluation of hepatic fibrosis during chronic hepatopathies is essential for patients care because it influences therapeutic decisions. Failure to perform liver biopsy in our patients did not allow us to determine the diagnostic performance of these various tests in the evaluation of hepatic fibrosis. It is therefore necessary to conduct further studies with liver biopsy to determine the diagnostic performance of these non-invasive tests in chronic HBV carrier in Togo.

\section{Compliance with ethical standards}

\section{Acknowledgments}

We appreciate the cooperation of the patients who agreed to participate in the study. Our thanks go to the COCHIN Hospital and the management of the Campus Teaching Hospital for the support with ideal environment to perform the study.

\section{Disclosure of conflict of interest}

All authors declare no conflict of interest 


\section{Statement of informed consent}

Informed consent was obtained from all individual participants included in the study.

\section{References}

[1] World Health Organization. Guidelines for the prevention, care and treatment of people with chronic hepatitis B infection. March 2015.

[2] Haute autorité de santé. Méthodes d'évaluation de la fibrose hépatique au cours des hépatopathies chroniques [internet]. Décembre 2006; 176.

[3] Haute autorité de santé. Méthodes non invasives de mesure de la fibrose hépatique. Diagnostic de la cirrhose non compliquée [internet]. Décembre 2008; 160.

[4] Wai C, Greenson JK, Fontana RJ, Kalbfleisch JD, Marrero JA, Conjeevaram HS, et al. A simple noninvasive index can predict both significant fibrosis and cirrhosis in patients with chronic hepatitis C. Hepatology. 2003; 38(2): 51826.

[5] Vallet-Pichard A, Mallet V, Nalpas B, Verkarre V, Nalpas A, Dhalluim-Venier V, et al. FIB-4: an inexpensive and accurate marker of fibrosis in HCV infection. Comparison with liver biopsy and fibrotest. Hepatology. 2007; 46(1): 32-5.

[6] Munteanu M. Non-invasive biomarkers fibrotest-actitest for replacing invasive liver biopsy: the need for change and action. Journal of gastrointestinal and liver Diseases. 2007; 26(2): 173-4.

[7] Mallet V, Dhalluim-Venier V, Roussin C, Bourlière M, Pettinelli ME, Giry C, et al. The accuracy of FIB-4 for the diagnostic of mild fibrosis in chronic hepatitis B. Alimentary Pharmacology and Therapeutics. 2009; 29(4): 40915.

[8] Lupşor M, Badea R, Stefănescu H, Grigorescu M, Sparchez Z, Serban A, et al. Analysis of histopathological changes that influence liver stiffness in chronic hepatitis C. Results from a cohort of 324 patients. Journal of gastrointestinal and liver Diseases Dis. 2008; 17(2): 155-63.

[9] Poynard T, De Ledinghen V, Zarski JP, Stanciu C, Munteanu M, Verginiol J, et al. Relative performances of fibrotest, fibroscan, and biopsy for the stage of liver fibrosis in patients with chronic hepatitis C: a step toward the truth in the absence of a gold standard. Journal of hepatology. 2012; 56(3): 541-8.

[10] Leroy V, Hilleret MN, Sturm N, Trocme C, Renversez JC, Faure P, et al. Prospective comparison of six non-invasive scores for the diagnosis of liver fibrosis in chronic hepatitis C. Journal of hepatology. 2007; 46(5): 775-82.

[11] Mahassadi A, Attia A, Bathaix F, Agbé N, Doffou S, Kissi H, et al. Diagnostic accuracy of biochemical markers of fibrosis in black African patients with chronic hepatitis B. Health. 2010; 2(12): 1413-20.

[12] Bonnard P, Sombié R, Lescure FX, Bougouma A, Guiard-Schmid JB, Poynard T, et al. Comparison of elastography, serum marker scores, and histology for the assessment of liver fibrosis in hepatitis B virus (HBV)-infected patients in Burkina Faso. American Journal of Tropical Medicine and Hygiene. 2010; 82(3): 454-8.

[13] Lawson-Ananissoh LM, Bouglouga O, Bagny A, Kaaga L, El Hadj Y, Redah D. Knowledge of hepatitis B virus among outpatients consulting in the hepatogastroenterology department at the Lome Campus University Hospital. Médecine et santé tropicales. 2015; 25(3): 319-22.

[14] Banla AK, Gani KT, Halatoko WA, Layibo Y, Akolly K, et al. Prevalence of the surface antigen of hepatitis B virus among youth aged 15 to 24 in Togo in 2010. Journal of infectious diseases and therapy. 2015; 3: 238.

[15] European Association for the Study of the Liver (EASL). Clinical Practice Guidelines on the management of hepatitis B virus infection. Journal of Hepatology. 2017; 67: 370-98. 